\title{
Priority-based parking system for university campus using IOT and CVRP
}

\author{
Marouane EL Midaoui ${ }^{1} *$, Mohammed Qbadou ${ }^{1}$, and Khalifa Mansouri ${ }^{1}$ \\ ${ }^{1}$ SSDIA Mohammedia, Morocco
}

\begin{abstract}
The significant increase of cars and the population growth due to urban area development are considered as a challenge for the smart city last years. Resulting to traffic congestion, especially in the parking lot. One of the city major impacted infrastructures is the university campus. A parking management system saves time and resolves the traffic congestion. However, the rapid growth inside the university is not taking into consideration by the current parking management system. Also, the universities and other institutions are a hierarchical organism and have a specific priority and roles to be respected for spaces which include parking lot. The current paper addresses the parking management system inside universities with respect of the requirements of institution and proposes architecture based on IoT system to insure the flowless of the traffic inside the university. Also, a hybrid VRP and MDVRP solution to provide a fair distribution of parking lots between different visitors while respecting the priorities.
\end{abstract}

\section{Introduction}

During the last decades, the number of vehicles in use has increased worldwide. Thus, parking has become a widespread challenge almost all cities [1]. Even if this problem can be observed whatever, it is clearer in canter of cities, open markets, shopping malls, hospitals, administrations, schools, and universities [2]. Parking areas can be divided into two categories: outdoor parking and indoor ones. Parking is managed using platforms that collect parking spots status and display that information to users [3]. However, not all drivers can share parking areas information even with local data silos [5] and data of isolated parking cannot be transmitted to a unified platform. Therefore, smart parking concept has been introduced to overcome parking difficulties and the leak of parking space management in smart cities. Smart parking is a major example of smart city application. Usually, drivers try to search for available parking spots driving around, only locating a parking spot based on their local knowledge and luck. This way of search for parking spot consumes both fuel and time, but there is no assurance to find an available parking place especially during high vehicle traffic times. One of the solutions is implement a system that shows unused parking spots to the vehicle and lets the driver chooses a free parking place. However, this approach is not suitable for all contexts, because it's made drivers to select by them. But also, the routed path to the chosen parking spot could be congested or far from the entrance, causing the parking spot to be occupied when the driver arrives, and also this kind of system do not take into account that each driver has its own needs or role in the infrastructure.
Existing systems connect sensors nodes to a management platform server using different types of wireless networks for communication [11]. This requires more gateways, increasing the complexity and making it hard to deploy and maintain scalability of smart parking. The development of smart parking systems is, then, severely constrained in most areas when necessary. Universities are not in rest. In fact, due to the increasing number of staff/students owning vehicles, campuses are more and more full of cars and parking spaces are getting more occupied than ever. Both students and staff are daily challenged to find a vacant parking space and can make many rounds in vain. They would waste -as result- precious energy and time and may be late for their classes and work. Hence, rather than conventional systems of parking management, IoT-based Management parking platforms may overcome such faced problems. Smart system may help vehicles owner within the campus in pollution reduction, time-saving and efficient resource utilization. These platforms are essential more than never not only for smart cities development, but also for the convenience of staff, students and administrations but also people with special needs. Recently, with the emergence of smart cities and the Fourth Industrial Revolution (IR 4.0), universities have devoted more time and focus to the smart campus paradigm and numerous initiatives have been launched to build smart campus projects with smart parking management. An IoT device fits into this context. IoT networks help sharing data about the outdoor parking areas of the campus over the Internet of security and management purposes. To build such platform multiple sensors will be needed in order to erform vehicle detection. The present paper present a parking

Corresponding author: marouane.elmidaoui@gmail.com 
management system using IOT technologies with implementation of hybrid VRP and MDVRP, this implementation is for supporting priority and urgency inside university campus. An important asset of the present solution remains the enhanced visual detection accuracy relaying on ultrasound sensor and raspberry Pi Camera, the utilization in any parking area within $\mathrm{Wi}-\mathrm{Fi}$ coverage and GPS signal and the BLYNK IoT available to all parking users. Besides the IOT side, the management of roles and priorities are provided by implementation of an hybrid VRP in case of the parking's services required this kind of features such as priority for university stuff, and people with special needs. Depending on the data to be collected and shared, it is question of multiple sensors (ultrasonic, Pi camera and GPS). The first will be placed in each parking spot, while the others can cover all parking slots. Higher efficiency of the platform, low price will be ensured by the use of Raspberry Pi, especially for large-scale smart campus deployments. With the embedded Wi-Fi interface, the Raspberry Pi can be used to connect to hotspot internet inside the campus and performs as the gateway of the IoT system. In order to get sensors data available via mobile apps, the collected data will be uploaded to the BLYNK IoT server.

\section{Related works}

Recently, the spaces around the cities and rural areas are being used up to build commercial zones and skyscrapers, especially with the development of the suburban area. Nevertheless, the available parking slots are always scarcity regardless of the size of the city. Because of this development, traffic congestion, limited car parking facilities, road safety and other problems were raised in terms of transportations in the city. It is due to these issues that many smart parking systems are established in the market. Although most of the parking systems presented in the literature provide solutions to the design and development of parking availability information system, parking reservation system, occupancy detection, and parking lot management, few works emphasize on the identification of the vehicle's clearance to access the parking space in the first place. With the emergence of the IoT paradigm, the creation of a parking management platform for smart campus is becoming more and more achievable. For 20 years, a smart living style has been promoted due to modern communication technologies and microcontrollers and IoT which is one of the main drivers of future smart spaces. The term IoT has been more inclusive, covering a wide range of applications like healthcare, utilities, transport, and so on [12]. Delot and al. [4] study the fairness of parking allocation in vehicular networks VANET. The study concludes that it is less clear which slot would be best, the authors propose dissemination protocols with an encounter probability parameter, the IoT unlocks new operational technologies of various applications and offers environmental benefits as well. Thanks to IoTs, spaces are not only 'smart', but intelligently connected too. Coming out with the same principles of smart city applications, the concept of smart campus is developed on the basis of digital campuses. Compared with the latter, the smart campus cuts operational costs, provides services in a timely manner, and reduces effort. Smart campus involves that the administration will adopt advanced technologies to provide high-quality services to the campus community and control and monitor facilities on campus automatically [13]. In the recent decades, many actions have been undertaken in order to improve campus services. Many universities worldwide have financed several projects in order to build a smart campus scheme involving multiple structures and sites and save energy consumption, reduce $\mathrm{CO} 2$ emissions, and rise sustainability and interaction. Concretely, in staff and student's everyday life, searching for parking space is more painful than ever and leads to develop traffic congestion and excess consumption of fuel [14] in campuses as well. Smart parking solutions have attracted considerable attention from both academia and industry. These provide information about the available parking lot systems by collecting real-time data on the available spaces in a parking area using the sensors scattered in the parking lot. Updated continuously, the result of that information will be displayed in the software application regarding the availability status of the parking lot and its location. According to some recently relevant studies on IoT- based smart parking systems, an 8-bit PIC16F73 CMOS FLASH-based microcontroller is used to read and send the data to the IoT web page through Wi-Fi [16]. This Expressive Systems Smart Connectivity Platform (ESCP) Wi-Fi module is used to connect the device to the Internet, while another IR proximity sensor is used to detect parking occupancy. The main limitation to be considered is the microcontroller complexity. It needs an extra module for $\mathrm{Wi}-\mathrm{Fi}$ connection and can admit a limited number of sensors. Another study proposed an IoT-based smart parking system using ultrasonic sensors connected wirelessly to an RPi via ESP8266 Wi-Fi chip [17]. This RPi is used for processing and transmitting sensor data to the IBM MQTT Server. The server save information such as parking entry time, parking duration, payment required, and payment mode so that users could access the information through their mobiles. However, the system relies on one sensor type which may affect data accuracy. Besides, it requires a subscription to the IBM MQTT server. Another related work considers two priorities parking for University of Murcia using Multiagent systems and Semantic Web technologies [15]. Of course, this study proposes an architecture based on Multi-agent systems (MAS) and Semantic Web technologies such as OWL and SWRL technologies to develop an intelligent parking management application, where agents interact to reach a consensus about the assignment of a parking area to avehicle. This work tackles as well the inherent problem related to the rise of conflicts in MAS by means of the integration of an argumentation system called ASBO into the proposed architecture. Furthermore, a study on management strategy of the on-street parking in Urban Residential Area, taking Harbin as example is mainly related to our 
work [7]. In fact, this study takes Harbin residential area as an example to explore and explore on- road parking management strategy. Firstly, the status of on-road parking in residential areas is examined, and the deficiencies of on-road parking management in residential areas are analysed. Referring to advance management experience and mode of on-road parking in residential areas abroad, and combined with the actual parking situation of Harbin residential areas, this paper puts forward the corresponding optimization strategy of on-road parking management. For the routing part, VRP natural computing algorithms show a suitable result. B. $\mathrm{Yu}$ and al. [8] proposed an improved ant colony algorithm to solve VRP which gives better results in the comparison between other heuristic methods, yet another approach proposed by Y. J. Gong et al. [9] based on PSO for VRP with time windows, the simulation result shows the efficiency of Solomon's benchmark testing algorithm. Multi-Depot Vehicle Routing Problem is considered one of the well-known solutions in the research field currently for solving the delivery of goods in multi- depot instead of one depot as VRP, and the goal is to optimize cost and exchange between multiple sources and destinations. In [10] S. Zhang and al. proposed a model MDGVRP (MD green VRP) based on an ant colony with a solution to route the optimal path while taking into account the capacity of the vehicle and also fuel capacity of such vehicle. Vehicle routing problems cannot be resolved with a height precision, caused by the height computing power. To get better precision, the genetic algorithm (GA) can be used in this case, because of the stochastic properties and efficiency of GA, MDVRP can be resolved by using those techniques. Author in [6], Osaba and al. describe several applied metaheuristic approaches. a Vehicle Routing Problem (VRP) is proposed with a relaxed priority rules (VRP- RPR) customer with the highest priorities need to be served before lower priority ones [18]. This research proposed a Mixed Integer Linear Programming (MILP) model to formulate the problem and to solve small-sized instances. A metaheuristic based on the Adaptive Large Neighbourhood Search (ALNS) algorithm with problemtailored components is designed to handle the problem at larger scales.

\section{Our contribution}

We proposed a VRP solution for resolving routing and scheduling system for the parking lot in a campus university, the approach is based on classical Capacitated VRP (CVRP) with a reformulation to take priority as capacity, and parking as customers, we consider two main components as depicted in Figure 1 the incoming car with assigned priority to use the routes and a parking lot with two different statuses full or free and a priority assigned. The entrance, in this case, is the center of the route where the incoming car is located (near to the dispatcher) or what is known in VRP as depot, all the parking in the system are targets. Each parking has a priority to match the incoming car, as shown at the left side of figure 1 , the incoming car with priority 1 match nearest parking with priority 1 when the parking is free, which mean in CVRP the vehicle with capacity 1 will serve at first the customer with demand of 1 unit. And in the case of none free or full parking lot, the incoming car will go the nearest parking lot with lower priority as shown in the right side of the figure 1 . There is also case of multiple entrances (or multiple dispatcher), in this case we will change the VRP to multi depot vehicle routing problem, but the same priority will be applied to the MDVRP by changing the Capacitated MDVRP to a priority based MDVRP.

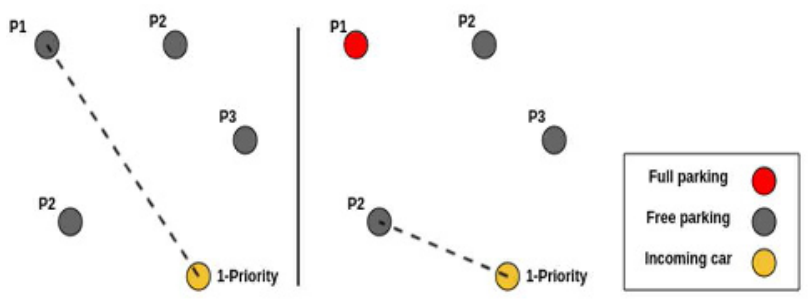

Fig. 1. Routing in case of free and full parking slot.

NB: The solution is not limited only to universities campus, this solution can be implemented also in any similar environment (markets, hospitals, malls, administrations, etc).

\subsection{The Vehicle Routing Problem}

The Vehicle Routing Problem (VRP) is a class of Operations research and combinatorial optimization methods. The aim is to determine the routes of a fleet of vehicles to deliver a list of customers or to conduct tours of interventions (maintenance, repairs, and checks) or visits (medical visits, commercial, etc.). The main idea is to minimize the cost of delivering goods. This approach is a classic extension of the traveling salesman problem. It is an employee who is responsible for distributing products (or services) on behalf of one or more employers, projecting this approach in our context, customers are translated to parking, a depot to the dispatcher. Figure 2 explains the routing from one dispatcher to parking using VRP.

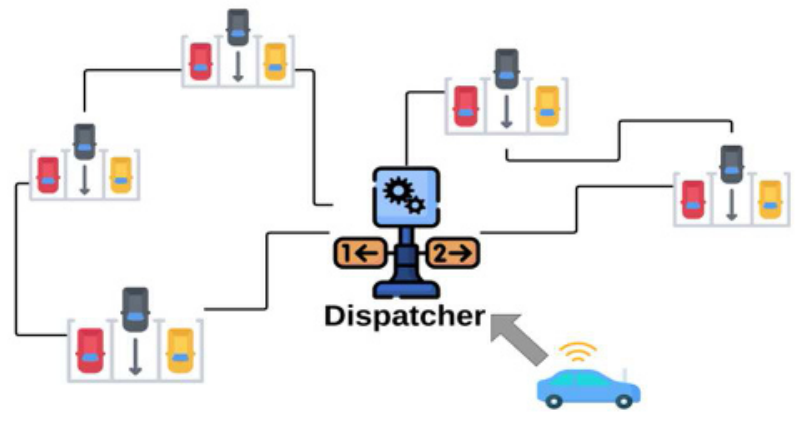

Fig. 2. Parking routing based on VRP.

Based on transportation network, the notations used for the formulation of the VRP are based on a simple graph $\mathrm{G}=(\mathrm{V}, \mathrm{E})$ with: 
$\mathrm{V}=\left\{\mathrm{V}_{0}, \mathrm{~V}_{1}, \ldots, \mathrm{V}_{\mathrm{n}}, \mathrm{V}_{\mathrm{n}+1}\right\}$ a set of vertices with $\mathrm{V}_{0}=$

$\mathrm{V}_{\mathrm{n}+1}$ representing the deposit and $\mathrm{V}^{\prime}=\mathrm{V} /$

$\left\{\mathrm{V}_{0}, \mathrm{~V}_{1}, \ldots, \mathrm{V}_{\mathrm{n}}, \mathrm{V}_{\mathrm{n}+1}\right\}$ the set of clients.

$E=\left\{\left(V_{i}, V_{j}\right) / V_{i}, V_{j} \in V^{\prime}, i \neq j\right\}$ is the set of edges forming the routes. Each tour starts at v0 and ends at vn+1.

A matrix of costs or distances $c_{i j}$ between customers $V_{i}$ and $\mathrm{V}_{\mathrm{j}}$, with $\left(\mathrm{C}_{0, \mathrm{n}+1}=0\right)$

$\mathrm{D}$ represents a vector of customer requests.

$\mathrm{R}_{\mathrm{i}}$ Is the tour of vehicle $\mathrm{i}$ represented by a permutation of customers visited by the vehicle.

The fleet of vehicles is made up of all identical vehicles with capacity $\mathrm{C}$ and fixed cost $\mathrm{F}$. A vehicle is assigned to each round.

This model contains noted decision variables $x_{\mathrm{ij}}^{\ell}$ (defined $\square i j \quad \mathrm{~V}, \square \ell \quad$ ) and take the value 1 if the vehicle $\ell$ goes from customer i to customer j, 0 otherwise. The VRP can therefore be formulated as follows:

$$
\begin{aligned}
& \operatorname{Min} Z=F \sum_{j} \sum_{V^{\prime} l} x_{0 j}^{1}+\sum_{i} \sum_{j} c_{i j} \sum_{1} x_{i j}^{1} \\
& \sum_{\mathrm{i} V} \sum_{\ell} \mathrm{x}_{\mathrm{ij}}^{\ell}=1 \\
& \sum_{\mathrm{j} \vee} \sum_{\ell} \mathrm{x}_{\mathrm{ij}}^{\ell}=1 \\
& \text { j V' } \\
& \text { i V' } \\
& \sum_{j V^{\prime}} x_{0 j}^{\ell}=1 \\
& \sum_{\mathrm{i} V \mathrm{ik}} \mathrm{x}_{\mathrm{ik}}^{\ell} \quad \sum_{\mathrm{j} \mathrm{V}} \mathrm{x}_{\mathrm{kj}}^{\ell}=0 \\
& \ell \\
& \text { k V', l } \\
& \sum_{\mathrm{i} \mathrm{V}^{\prime}} \mathrm{x}_{\mathrm{i}, \mathrm{n}+1}^{\ell}=1 \\
& \sum_{i V^{\prime}} d_{i} \sum_{j \in V} x_{i j}^{\ell} \quad C \\
& \ell
\end{aligned}
$$

The objective function (1) indicates the total cost to be minimized and which includes the cost of vehicles and the cost of rounds. The constraints (2) and (3) indicate that each customer must be assigned to exactly one vehicle. The constraints (4), (5) and (6) model the flow constraints which guarantee that each vehicle leaves the depot, and then visits at least one customer to finally return to the depot. The constraint (7) indicates that the capacity of the vehicles must not be exceeded.

\subsection{Multi Depot Vehicle Routing Problem}

MDVRP is a VRP with Multi depots from which the vehicle can serve its customers - in our case parking-. If parking is grouped around dispatchers, then the distribution problem must be modelled as a set of independent VRPs as shown above. However, if parking and dispatcher are intertwined or for specific needs the delivery must be from another dispatcher, the problem of multi-depot vehicle routing needs to be resolved. The MDVRP processes several deposits. Each vehicle or set of vehicles is assigned to a single depot, which is usually both the origin and destination of the vehicle route. The figure 3 presents the multiple dispatcher deliveries for a set of parking.

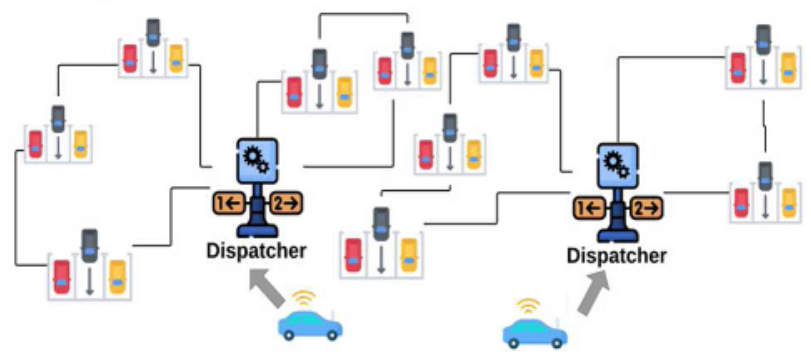

Fig. 3. Parking routing based on MDVRP.

We can present our MDVRP mathematically as follows: $\mathrm{G}=(\mathrm{V}, \mathrm{E})$ is a graph where $\mathrm{V}$ is the set of vertices partitioned into two collections. $V_{c}=\left\{V_{1}, V_{2}, \ldots, V_{n}\right\}$ Represent the list of the parking. $V_{p}=$ $\left\{V_{n+1}, V_{n+2}, \ldots, V_{n+m}\right\}$ represent the collection of dispatcher, E set of edges connecting two points. A Cost matrix $D=\left(D_{i j}\right)$ is the length of $\operatorname{arc}\left(v_{i}, v_{j}\right)$ (corresponding to distance). Expecting D symmetry, and the distance between sets of three points satisfies the triangle inequality. Each dispatcher $\mathrm{V}_{\mathrm{n}+\mathrm{m}} \in \mathrm{V}_{\mathrm{d}}$ has the same model. $\mathrm{k}_{\mathrm{m}}$ (The number of vehicles at the dispatcher) are identical in term of vehicle (model, capacity Q, shape).

Each vehicle starts from a dispatcher, serving a set of parking, at the end of the route or when $\mathrm{Q}$ is not enough to serve the next Parking, the vehicle must return to the starting dispatcher to refill. Every parking is visited only once by one vehicle, we consider in this example that a route can be done only by one vehicle. The total demand for each route does not exceed the vehicle capacity $Q$. The vehicle cannot exceed the vehicle capacity $Q$, and the number provided by each dispatcher is limited.

Dispatchers cannot route each other's (dispatcher cannot route to another dispatcher). The main objective for MDVRP, in this case, is to link parking into the proper dispatcher with minimization of distance travelled by vehicle across the network. $\mathrm{n}$ parking are grouped to form $m$ clusters. Clusters consist of $n_{1}, n_{2}, \ldots, n_{m}$ number of parking. The number of vehicles based at a dispatcher is $\mathrm{k}_{\mathrm{m}}$. $\mathrm{k}_{\mathrm{j}}$ is the group of parking linked to a vehicle. MDVRP formulation for finding $\mathrm{x}$ which is the minimize can be described as following:

$$
\begin{aligned}
& \text { Decision variables } \mathrm{X} \text { and } \mathrm{Y} \text { : } \\
& \mathrm{x}_{\mathrm{ijkm}}=\{\text { parking } \mathrm{j} \text { else } 0\} \\
& \mathrm{y}_{\mathrm{ikm}}=\{\text { dispatcher } \mathrm{m} \text { else } 0\} \\
& \mathrm{MIN}=\sum_{\mathrm{p}=1}^{\mathrm{m}} \sum_{\mathrm{q}=1}^{\mathrm{k}_{\mathrm{p}}} \sum_{\mathrm{i}=1}^{\mathrm{n}} \sum_{\mathrm{j}=1}^{\mathrm{n}} \mathrm{d}_{\mathrm{ij}} \mathrm{x}_{\mathrm{ijqp}} \\
& \sum_{\mathrm{i}=1}^{\mathrm{n}} \mathrm{q}_{\mathrm{i}} \mathrm{y}_{\mathrm{iqp}} \leq \mathrm{Q} \\
& 0 \leq \mathrm{n}_{\mathrm{jq}} \leq \mathrm{n}_{\mathrm{j}} \\
& \sum_{\mathrm{q}=1}^{\mathrm{k}_{\mathrm{j}}} \mathrm{n}_{\mathrm{jq}}=\mathrm{n}_{\mathrm{j}} \forall \mathrm{j}=1 \mathrm{~m} \\
& \sum_{\mathrm{j}=1}^{\mathrm{m}} \mathrm{n}_{\mathrm{j}}=\mathrm{n} \\
& \sum_{\mathrm{p}=1}^{\mathrm{m}} \sum_{\mathrm{q}=1}^{\mathrm{k}_{\mathrm{p}}} \mathrm{y}_{\mathrm{iqp}}=1 \\
& \mathrm{X}_{\mathrm{ijqp}}=1 \mathrm{~V} 0 \\
& \mathrm{y}_{\mathrm{iqp}}=1 \mathrm{~V} 0
\end{aligned}
$$


The objective function according to Equation (8) minimizes the total delivery distance and cost of each vehicle within a dispatcher. (9) limits capacity Q of the vehicle, (10) indicates the parking served by each vehicle must not overtake the number of the parking a dispatcher can deliver to. (11) Shows the sum of the parking served by the whole route should be the sum of the parking served by the dispatcher. (12) shows every parking served by a dispatcher. (13) Guarantee that every parking must be visited only once by a vehicle. (14) (15) is the value bound of the decision variables.

\subsection{Device Hardware Components}

Raspberry Pi 4 model B:

- Broadcom BCM2711, quad-core Cortex-A72 (ARM v8)64-bit SoC @1.5GHz

- 4GB LPDDR4-2400 SDRAM

- $\quad 2.4 \mathrm{GHz}$ and 5. $0 \mathrm{GHz}$ IEEE 802. 11B/g/n/ac Wireless LAN, Bluetooth 5. 0, double-true Gigabit Ethernet

- $2 \times$ USB 3.0 ports, 2 x USB 2. 0 Ports

- $\quad 2 \times$ micro HDMI ports supporting up to $4 \mathrm{Kp} 60$ video resolution

- 2-lane MIPI DSI/CSI ports for camera and display

- 4-pole stereo audio and composite video port

- Micro SD card slot for loading operating system and data storage

- $\quad$ Requires 5. 1V, 3a power via USB Type C or gpiopoep (power over Ethernet)

Pi camera :

- 8 megapixel camera capable of taking photographs of $3280 \times 2464$ pixels

- $\quad$ Capture video at 1080p30, 720p60 and 640x480p90 resolutions

- All software is supported within the latest version of Raspbian Operating System

- Applications: CCTV security camera, motion detection, time lapse photography

- For Use With: Compatible with all Models of Raspberry Pi 1, 2 \& 3

- Kit Contents: Raspberry Pi Camera Board, Ribbon Cable

HSC-SR04 ultrasonic sensor:

- Operating voltage: $+5 \mathrm{~V}$

- Theoretical Measuring Distance: $2 \mathrm{~cm}$ to $450 \mathrm{~cm}$

- Practical Measuring Distance: $2 \mathrm{~cm}$ to $80 \mathrm{~cm}$

- Accuracy: $3 \mathrm{~mm}$

- Measuring angle covered: $<15^{\circ}$

- Operating Current: $<15 \mathrm{~mA}$

- Operating Frequency: $40 \mathrm{~Hz}$

NEO-6 M GPS module:

- $\quad$ Receiver Type : 50 channels, GPS L1(1575.42Mhz)

- Horizontal Position Accuracy : $2.5 \mathrm{~m}$

- Navigation Update Rate : $1 \mathrm{HZ}$ (5Hz maximum)

- Capture Time : Cool start: 27sHot start: 1s

- Navigation Sensitivity : $-161 \mathrm{dBm}$

- Communication Protocol : NMEA, UBX Binary, RTCM
- $\quad$ Serial Baud Rate : 4800-230400 (default 9600)

- Operating Temperature : $-40^{\circ} \mathrm{C} \sim 85^{\circ} \mathrm{C}$

- Operating Voltage : $2.7 \mathrm{~V} \sim 3.6 \mathrm{~V}$

- Operating Current : $45 \mathrm{~mA}$

- TXD/RXD Impedance : $510 \Omega$

SOFTWARE INVOLVED

- Raspbian

- BLYNK

- Proteus Design Suite

- $\quad$ Siemens NX 10

- VNC Viewer

As shown in Figure 4 based on the hardware connected components. Sensors, GPS shield and Camera record data. Frist, the GPS module provides the exact location for the parking area. Then the ultrasonic sensors, sense the vehicle presence inside the parking spot. The whole data is then trigged to the Raspberry Pi. The data is uploaded to BLYNK server to be available on mobile IOS and Android smartphones. Drivers can connect their smartphones and access the information about parking slots occupancy. If required, visual monitoring is also available thanks to Camera connected and other users may be content to observe and monitor the parking area via video streaming. A real-time condition of the parking area is streaming to the cloud for monitoring. Also, the camera can be used to security matters and ensure security surveillance of parking area.

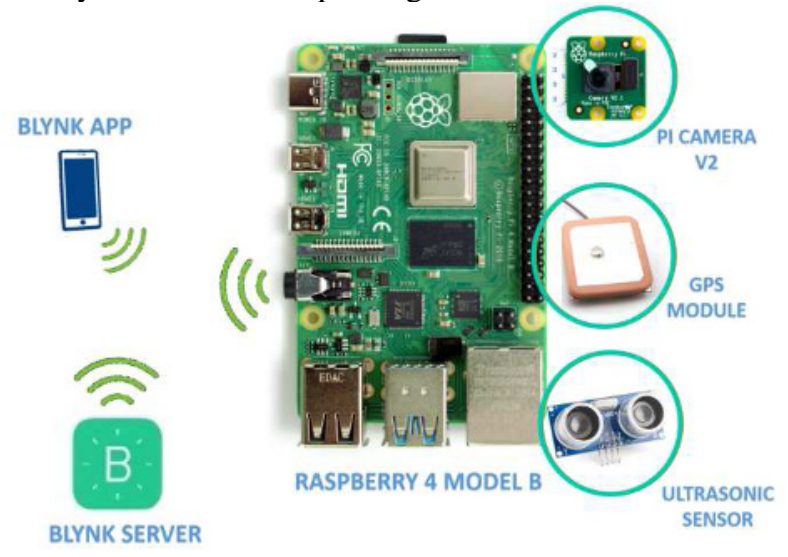

Fig. 4. IOT architecture of raspberry pi smart parking system.

Concretely, and to ensure the connection between these devices, Raspberry Pi GPIO pins are connected to the input/output pins of the sensors (ultrasonic sensor, GPS sensor, and Pi camera). Since the HSC-SR04 is used to detect the presence of a car in the slot, it changes the LED position ON/OFF according to the status of the slot. Depending on the data to be collected and shared, it is question of multiple sensors (ultrasonic, Pi camera and GPS). The first will be placed in each parking spot, while the others can cover all parking slots. Higher efficiency of the platform, low price will be ensured by the use of Raspberry Pi, especially for large-scale smart campus deployments. With the embedded Wi-Fi interface, The Raspberry Pi can be used to connect to hotspot internet inside the campus and performs as the gateway of the IoT system. In order to get sensors data 
available via mobile apps, the collected data will be uploaded to the BLYNK IoT server.

\section{Computational experiments and results}

In this section we perform computational experiments to validate the CVRP approach in comparison with a random selection of the parking lot for each vehicle. The simulation was in several contexts. First one is a blind priority parking, which is the regular parking without any priority for staff or students with special needs. The second context was with a role and priority based in the campus. And third context is with multi-size parking spots for cars, truck, bus, or motorcycle As shown in the figure 5, the total of meters driven by all vehicles in the first context for each approach is very similar but the second solution was ahead with only 10 meters. In the second context the CVRP based parking system outperformed the second one with more than $21 \%$ overall (1428 meters for CVRP in comparison with 1194 meters for random), however these results are not fixed, due to the randomized aspect of the second solution. The third context was not suitable for the CVRP based solution, because the system was not designed to perform in that form of parking.

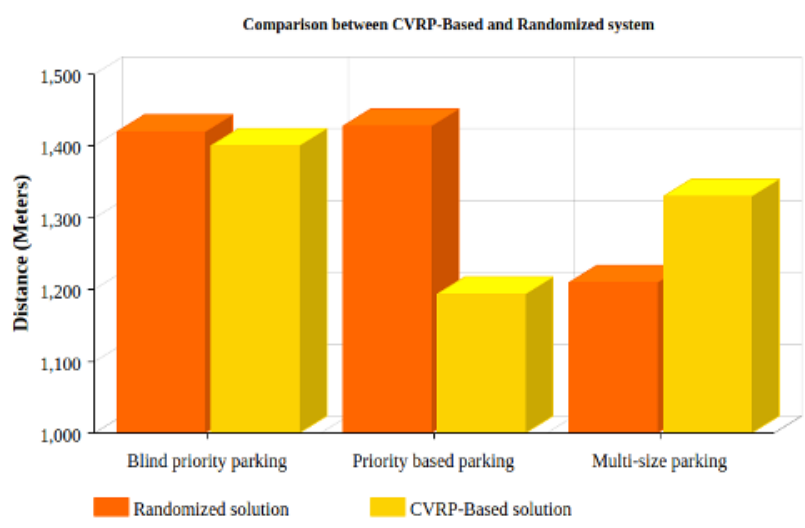

Fig. 5. Comparison between CVRP and randomized system.

The CVRP based parking solution is suitable in infrastructure that support or required a hierarchical or humanitarian priority in comparison with randomized solution

\section{Conclusion}

Searching for available parking lots inside campus emerges as one of the major impacts for student lateness inside the universities. The unorganized searching of parking lots can cause congestion, negative environmental effects. the proposed solution addresses these issues with respecting requirements inside the university, Staff/students/visitors can easily monitor the parking lot around the campus or be dispatched using the CVRP to place each priority in a proper lot. it has been proved is the simulation that the current solution is weak in case of multi-size parking lots, however this kind or parking are rare, this model represents the tool for assigning parking lots to vehicles within a dynamic configuration with respecting the priority and needs of each case, it can be applied in different structure that has a similar requirement. It should be noticed that the model can be improved by adding the multi-size to the chromosome structure before routing.

\section{References}

1. C.G. Hoehne, M.V. Chester, A.M. Fraser, D.A. King, Valley of the sun-drenched parking space: the growth, extent, and implications of parking infrastructure in Phoenix, Cities 89 (2019) 186-198.

2. J. Arellano-Verdejo, F. Alonso-Pecina, E. Alba, A. Guzmán Arenas, Optimal allocation of public parking spots in a smart city: problem characterisation and first algorithms, J. Exp. Theor. Artif. Intell. 31 (4) (2019) 575-597.

3. F. Al-Turjman, A. Malekloo, Smart parking in IoTenabled cities: a survey, Sustain. Cities Soc. (2019) 101608.

4. Delot, Thierry, Ilarri, Sergio, Lecomte, Sylvain, \& Cenerario, Nicolas. 2013. Sharing with caution: Managing parking spaces in vehicular networks. Mobile Information Systems, 9(1), 69-98.

5. J. Liu, J. Wu, L. Sun, Control method of urban intelligent parking guidance system based on Internet of Things, Comput. Commun. 153 (2020) 279-285.

6. Osaba, E., Yang, X. S., \& Del Ser, J. (2020). Is the vehicle routing problem dead? an overview through bioinspired perspective and a prospect of opportunities. Nature-Inspired Computation in Navigation and Routing Problems, 57-84.

7. Xiaoyan, Zhu \& Xiqiao, Zhang \& Xu, Sun. (2021). Study on management strategy of the on-street parking in Urban Residential Area - Taking Harbin as an Example. IOP Conference Series: Earth and Environmental Science. 687. 012190. 10.1088/17551315/687/1/012190.

8. B. Yu, Z. Z. Yang, and B. Yao, "An improved ant colony optimization for vehicle routing problem," Eur. J. Oper. Res., vol. 196, no. 1, pp. 171-176, 2009, doi: 10.1016/j.ejor.2008.02.028.

9. Y. J. Gong, J. Zhang, O. Liu, R. Z. Huang, H. S. H. Chung, and Y. H. Shi, "Optimizing the vehicle routing problem with time windows: A discrete particle swarm optimization approach," IEEE Transactions on Systems, Man and Cybernetics Part C: Applications and Reviews, vol. 42, no. 2.10. pp. 254-267, Mar. 01, 2012, doi: 10.1109/TSMCC.2011.2148712.

10. S. Zhang, W. Zhang, Y. Gajpal, and S. S. Appadoo, "Ant Colony Algorithm for Routing Alternate Fuel Vehicles in Multi-depot Vehicle Routing Problem," 2019, pp. 251-260.

11. R. Iqbal, T. Maniak, C. Karyotis, Intelligent remote monitoring of parking spaces using licensed and 
unlicensed wireless technologies, IEEE Netw. 33 (4) (2019) 23-29.

12. H. Sundmaeker, P. Guillemin, P. Friess, S.J.C.o.E.R.P.o.t.I.o.T. Woelfflé, European Commision, "Vision and challenges for realising the Internet of Things," 3 (3) (2010) 34-36.

13. A. Abuarqoub, et al., A survey on internet of things enabled smart campus applications, in: Proceedings of the International Conference on Future Networks and Distributed Systems, ACM, 2017, p. 50.

14. E.C. Thangam, M. Mohan, J. Ganesh, and C.J.I.J.o.A.E.R. Sukesh, "Internet of Things (IoT) based Smart Parking Reservation System using Raspberry-pi," vol. 13, no. 8, pp. 5759-5765, 2018. Science and Engineering, 190, IOP Publishing, 2017.

15. Muñoz, A., \& Botía, J. A. (2010). Developing an Intelligent Parking Management Application Based on Multi-agent Systems and Semantic Web Technologies. Hybrid Artificial Intelligence Systems, 64-72. doi:10.1007/978-3-642-13769-3_8.

16. A. Kianpisheh, N. Mustaffa, P. Limtrairut, P. Keikhosrokiani, Smart parking system (SPS) architecture using ultrasonic detector, Int. J. Softw. Eng. Appl. 6 (3) (2012) 55-58.

17. A. Khanna, R. Anand, IoT based smart parking system, in: 2016 International Conference on Internet of Things and Applications (IOTA), IEEE, 2016, pp. 266-270.

18. Doan, Thanh \& Bostel, Nathalie \& Hà, Minh.(2021). The Vehicle Routing Problem with relaxed priority rules. EURO Journal on Transportationand Logistics. 10. 100039.10.1016/j.ejtl.2021.100039. 